03

\title{
Исследования рассеяния и рефракции света в водных дисперсных системах детонационного алмаза
}

\author{
(C) О.С. Везо, А.В. Войтылов, В.В. Войтылов, М.П. Петров ", А.А. Трусов \\ Санкт-Петербургский государственный университет, физический факультет, \\ 199034 Санкт-Петербург, Россия \\ Te-mail: m.p.petrov@spbu.ru
}

Поступила в редакцию 26.06.2018 г.

Использованы классические методы для исследования водных дисперсных систем алмаза. Дисперсная фаза, полученная детонационным методом, в процессе седиментации разделилась на восемь фракций. Согласно данным рентгеноструктурного анализа, все фракции содержали алмаз и графит. Методами динамического светорассеяния и электрооптики определены функции распределения частиц и их агрегатов по размерам в дисперсных системах, содержащих частицы данных фракций. Показано, что основное влияние на показатель преломления оказывают частицы, много меньшие длины световой волны, а частицы, соизмеримые с ней, существенно меняют мутность исследованных дисперсных систем, практически не влияя на их показатель преломления. Показано, что молекулярно-оптический расчет инкремента показателя преломления систем, содержащих частицы данных фракций, согласуется с результатами его экспериментального определения, если отношение размеров частиц к длине световой волны не превышает 0.1. Это позволяет определить доли алмаза и графита в дисперсной фазе детонационного алмаза. Значения инкремента показателя преломления исследованных систем, определенные согласно теории светорассеяния Ми, несколько выше экспериментальных значений. Показано, что теория рассеяния света сферическими частицами может быть использована при расчете индикатрис светорассеяния дисперсными системами детонационного алмаза.

DOI: $10.21883 /$ OS.2018.12.46938.181-18

\section{Введение}

Рефракция света - это хорошо известная и широко используемая характеристика молекулярных растворов $[1,2]$, а при исследовании дисперсных систем, в частности коллоидов, используется значительно реже $[3,4]$. Если для систем с частицами, близкими по размерам к молекулам, нет принципиальных сложностей при теоретическом описании рефракции, то в дисперсных системах, содержащих более крупные плотные частицы, такое описание становится сложным, а экспериментальное определение ее возможно только в тонких слоях и в случае крайне низкой концентрации частиц в системах. При увеличении размеров частиц существенно возрастает интенсивность диффузного некогерентного рассеяния, которое способно существенно повлиять на целый ряд оптических свойств. Так, было показано, что в водных дисперсных системах алмаза и графита с частицами 450 и $600 \mathrm{~nm}$ соответственно наблюдаются по методике электрического двойного лучепреломления ярко выраженные электрооптические эффекты. Однако показатель преломления таких дисперсных систем мало отличим от показателя преломления воды [4], что исключало появление двойного лучепреломления большой величины, обусловленного ориентационной упорядоченностью частиц в поле. Рефрактометрические исследования дисперсных систем такого типа малоэффективны. Однако для целого ряда дисперсных систем исследование рефракции позволяет получать надежные результаты о частицах меньших размеров. В данной работе предпринята попытка исследования рефракции дисперсных систем, содержащих частицы алмаза, полученных детонационным способом. Такие частицы перспективны для использования в медицине и промышленности.

\section{Рефракция света в нанодисперсных системах}

Дисперсные системы содержат не менее двух фаз вещества, в которых вещество хотя бы одной из фаз раздроблено. Такие системы, как правило, оптически неоднородны. При теоретическом описании показателя преломления таких систем возможны два подхода: молекулярно-оптический и основанный на рассеянии света отдельными частицами. Если частицы много меньше длины световой волны, то возможно рефракцию света дисперсных систем рассчитывать так же как и растворов, пренебрегая диффузным рассеянием света в них. В случае более крупных частиц используется иной подход, основанный на особенностях рассеяния света частицами. В данной работе сопоставляются два подхода определения инкремента показателя преломления нанодисперсных систем алмаза, содержащих частицы разных размеров.

\section{Молекулярно-оптическое приближение}

Удельную рефракцию дисперсной системы $R$, содержащей малые слабо рассеивающие свет частицы, можно определить, если известна объемная доля частиц $\Delta V / V$, 
а также рефракции вещества частиц (дисперсной фазы) $R_{p}$ и окружающей их молекулярной среды (дисперсионной среды) $R_{0}$. В этом случае по аналогии с молекулярными растворами можно записать

$$
R=R_{0}\left(1-\frac{\Delta V}{V}\right)+R_{p} \frac{\Delta V}{V}
$$

Согласно соотношению Лорентц-Лоренца [5],

$$
R=\frac{n^{2}-1}{n^{2}+2}, \quad R_{0}=\frac{n_{0}^{2}-1}{n_{0}^{2}+2}, \quad R_{p}=\frac{n_{p}^{2}-1}{n_{p}^{2}+2}
$$

где $n, n_{0}$ и $n_{p}$ - показатели преломления дисперсной системы, дисперсионной среды и дисперсной фазы. Объемную долю дисперсной фазы можно выразить через плотности дисперсной системы $\rho$, дисперсной фазы $\rho_{0}$, дисперсионной среды $\rho_{p}$ соотношением

$$
\frac{\Delta V}{V}=\frac{\rho-\rho_{0}}{\rho_{p}-\rho_{0}} .
$$

Если полагать, что поляризующее частицу поле определяется согласно теории Онзагера, а не Лорентца [5], как это представлено соотношением (2), то входящее в (1) значение $R_{p}$ следует умножить на коэффициент $k$, который для слабо вытянутых или сплюснутых частиц можно рассчитать по формуле

$$
k=3 \frac{4 n_{0}^{2}+5 n_{0}^{2} n_{p}^{2}-2 n_{p}^{2}+2}{\left(2 n_{0}^{2}+1\right)\left(n_{0}^{2}+2\right)\left(n_{p}^{2}+2\right)} .
$$

В случае частиц алмаза в воде $k=1.09$. Учитывая (1)-(3), а также при малой объемной доле частиц полагая $\left(n^{2}+2\right) /\left(n_{0}^{2}+2\right) \approx 1$, можно записать

$$
\frac{n^{2}-n_{0}^{2}}{\rho-\rho_{0}}=\frac{n_{0}^{2}+2}{\rho_{p}-\rho_{0}}\left(R_{p}-R_{0}\right) .
$$

Частица может иметь сложную структуру и состоять из нескольких компонент. В этом случае $R_{p}$ определяется как сумма удельных рефракций отдельных компонент, помноженных на их объемные доли. Плотность такой частицы $\rho_{p}$ определяется через плотности и объемные доли компонент аналогичным образом. При низких концентрациях дисперсной фазы в дисперсной системе, когда зависимости показателя преломления и плотности системы от концентрации $C_{p}$ частиц линейны, левую часть (4) можно выразить через инкременты $d n / d C_{p}$ и $d \rho / d C_{p}$, измеряемые экспериментально. В этом случае можно записать

$$
\frac{n^{2}-n_{0}^{2}}{\rho-\rho_{0}}=2 n_{0} \frac{d n}{d \rho}=2 n_{0} \frac{d n / d C_{p}}{d \rho / d C_{p}} .
$$

Соотношения (1), (2) и (4) следует рассматривать, как приближенные, и рамки их применимости такие же, как и в случае релеевского рассеяния.

\section{Однократное светорассеяние}

Показатель преломления дисперсной системы, содержащей частицы с размерами в несколько десятков нанометров и более и существенно рассевающие свет даже при их низкой концентрации, может быть получен как одно из следствий, вытекающих из теории однократного рассеяния света на частицах [6]. Характеристики света, рассеянного частицами с высоким показателем преломления, которым обладает алмаз, существенно зависят от размера и формы частиц, а также от ориентации частиц по отношению к волновому вектору и вектору электрического поля падающей на частицу световой волны. При определении показателя преломления коллоида, содержащего такие частицы, следует использовать элементы матрицы рассеяния частиц [7]. Построение матрицы рассеяния в большинстве случаев - крайне сложная задача. В исследованных в данной работе дисперсных системах рассеивающие свет частицы алмаза не имели четко выраженную форму, что позволило предполагать, что усредненные характеристики волны рассеянного такими частицами света и сферами близки. В рамках данного предположения применима теория рассеяния Ми [9], а вместо матрицы рассеяния можно использовать амплитудную функцию [6,7], которую можно рассчитать. Однако возможность использования данного предположения потребовало экспериментальной проверки, результаты которой представлены ниже.

Следует заметить, что прямая задача светорассеяния частицами произвольных размеров и формы может быть решена методом Т-матриц [8]. В данной работе рассматривается обратная задача определения показателя преломления дисперсной системы по их характеристикам светорассеяния.

В рамках приближения, учитывающего только электрическое поле световой волны, комплексный показатель преломления монодисперсного коллоида с низкой объемной долей сферических частиц связан с амплитудной функцией рассеяния частицы соотношением [7]

$$
\frac{n-i n^{\prime}}{n_{0}-i n_{0}^{\prime}}=1-i 2 \pi \frac{N_{p}}{k^{3}} S(0) .
$$

Здесь $k=2 \pi n_{0} / \lambda, \lambda$ - длина световой волны в вакууме, $S(0)$ - комплексная амплитудная функция рассеяния света сферами под нулевым углом, значения $n$ и $n^{\prime}$ соответствуют действительной и мнимой частям показателя преломления дисперсной системы, а $n_{0}$ и $n_{0}^{\prime}-$ дисперсионной среды. Учитывая (3) при определении счетной концентрации частиц $N_{p}$, из формулы (6) можно получить соотношения

$$
\begin{aligned}
\frac{d n}{d \rho} & =\frac{3 A}{2 k^{3} a^{3}\left(\rho_{p}-\rho_{0}\right)}, \\
\frac{d n^{\prime}}{d \rho} & =\frac{3 B}{2 k^{3} a^{3}\left(\rho_{p}-\rho_{0}\right)} .
\end{aligned}
$$

Здесь

$$
A=n_{0} \operatorname{Im}\{S(0)\}-n_{0}^{\prime} \operatorname{Re}\{S(0)\},
$$




$$
B=n_{0}^{\prime} \operatorname{Im}\{S(0)\}-n_{0} \operatorname{Re}\{S(0)\},
$$

$a$ - радиус рассеивающих свет сфер, а значение $n^{\prime}$ характеризует экстинкцию, которая зависит как от поглощения, так и рассеяния света частицами.

Соотношения (6)-(8) обоснованы, если размеры частиц невелики, и они находятся в однородном поле световой волны. При описании рассеяния света такими частицами магнитное поле световой волны не учитывается $[6,7]$. Если размеры частиц соизмеримы с длиной световой волны или превышают ее, то связать векторы напряженности и смещения электрического поля, входящие в уравнения Максвелла, возможно, если учитывать магнитное поле волны. В этом случае используется нелокальное приближение, согласно которому взаимосвязаны средние значения плотности тока, электрического и магнитного полей [10]. Методом Т-матриц может быть получено соотношение, связывающее показатель преломления дисперсной системы с амплитудной функцией $S(0)$ более общим, чем $(6)$, соотношением $[10,11]$

$$
\frac{n-i n^{\prime}}{n_{0}-i n_{0}^{\prime}}=\sqrt{1-2 i \frac{3}{2 k^{3} a^{3}} S(0) \frac{\Delta V}{V}} .
$$

Согласно (6) и (9), увеличение размеров частиц при неизменной их объемной доле приводит к понижению показателя преломления дисперсной системы. Как показывает расчет [11], в средах с поглощающими свет частицами и высоком показателем преломления показатель преломления дисперсной системы $n$ может принимать значения, которые меньше, чем значения показателя преломления дисперсионной среды $n_{0}$.

При определении показателя преломления и его инкремента в полидисперсных системах необходимо учитывать функцию распределения $\varphi(r)$ частиц по размерам $r$. В этом случае в соотношении (6) следует произвести замену произведения $N_{p} S(0)$ на величину $\left\langle N_{p} S(0)\right\rangle$, которая определяется как

$$
\left\langle N_{p} S(0)\right\rangle=\frac{3}{4 \pi} \frac{\Delta V}{V} \int \frac{S(0, r)}{r^{3}} \varphi(r) d r .
$$

\section{Техника эксперимента}

Для исследованных в данной работе дисперсных систем, подлежащих рефрактометрическим исследованиям, наряду с измерениями показателей преломления измерялись плотности с целью определения объемной доли дисперсной фазы. Изучалось рассеяние света в системах методами статического и динамического рассеяний и проводились электрооптические исследования с целью определения функции распределений частиц по размерам, установления их счетных концентраций. Исследования статического рассеяния света дисперсными системами также использовано для определения того, какие соотношения допустимы для теоретического описания показателя преломления коллоидов.

\section{Рентгеноструктурные и электронно-микроскопические измерения}

Рентгеноструктурный анализ дисперсной фазы проводился при помощи дифрактометра R-axis Rigaku методом Гандольфи. Для получения электронномикроскопических снимков был использован сканирующий микроскоп Zeiss Supra 40VP.

\section{Измерение показателя преломления дисперсных систем}

Так как показатель преломления в дисперсных системах - это усредненная характеристика, то следует использовать разные способы его измерения и убедиться в независимости результатов измерения от используемого способа. Нами были использованы три классических метода измерений, а концентрация частиц в дисперсных системах варьировалась. Первые два метода связаны с измерением угла полного внутреннего отражения (рефрактометр Abbemat WR/MW, точность измерения $\left.4 \cdot 10^{-5} \mathrm{nD}\right)$ и угла преломления скользящего луча (самодельный рефрактометр типа Пульфриха, точность измерения которого $2 \cdot 10^{-5} \mathrm{nD}$ ). Третий метод (интерферометрический) использован для определения разности показателей преломления дисперсной системы и чистой дисперсионной среды (интерферометр ITR-2, точность измерения $\left.1 \cdot 10^{-6} \mathrm{nD}\right)$. Первые два метода имеют меньшую точность, чем третий, но позволяют проводить исследования при более высоких объемных долях дисперсной фазы.

\section{Определение объемной доли частиц и изменение степени дисперсности}

Для определения объемной доли частиц использовано соотношение (3), плотность $\rho_{p}$ задавалась, а плотности $\rho$ и $\rho_{0}$, разность которых мала, измерялись с точностью $5 \cdot 10^{-6} \mathrm{~g} / \mathrm{cm}^{3}$ (прибор DMA 5000). Для отделения дисперсионной среды от частиц, а также получения систем, содержащих только мелкие частицы, использована ультрацентрифуга (Laboratory centrifuge Sigma 1-14), центробежное поле которой достигало $25000 \mathrm{~g}$.

\section{Определение индикатрис светорассеяния, размеров частиц и их агрегатов}

Электронно-микроскопические снимки пригодны для анализа размеров и формы частиц, но не позволяют анализировать взвешенные в дисперсной системе агрегаты из них. Для определения размеров частиц и агрегатов из них в дисперсной системе и анализа полидисперсности были использованы метод динамического светорассеяния (DLS) и электрооптический (EO) метод. Метод статического светорассеяния использован для определения индикатрис светорассеяния. С помощью прибора Photocor Complex измерялись индикатрисы светорассеяния и автокорреляционные функции интенсивности 
светорассеяния. Полагалось, что частицы, создающие флуктуации интенсивности светорассеяния, малы, а для определения размеров частиц и функции распределения по ним использовано соотношение

$$
G_{V}^{V}(t)=\int \exp \left(-t / \tau_{1}\right) \Delta I_{V}^{V} \varphi(r) d r
$$

в котором не учитывалось вращательное движение частиц.

Здесь автокорреляционная функция $G_{V}^{V}$ соответствует вертикальной поляризации падающего и рассеянного лучей. Определялась зависимость $f_{1}(r)=\Delta I_{V}^{V} \varphi(r)$, соответствующая функции распределения частиц и агрегатов по размерам $\varphi(r)$ с оптическим весом $\Delta I_{V}^{V}$. Оптический вес $\Delta I_{V}^{V}$ пропорционален $r^{6}$ и не зависит от угла рассеяния $\theta$ для частиц и агрегатов, малых по сравнению с длиной световой волны $\lambda$. В этом случае $\tau_{1}=1 / D_{T} q^{1}, q=k \sin (\theta / 2), k=2 \pi / \lambda, D_{T}$ - константа поступательной диффузии частиц или агрегатов [12]. Для частиц, соизмеримых с длиной световой волны или больших ее, зависимость $\Delta I_{V}^{V}$ от $r$ может быть сложной и немонотонной, а $G_{V}^{V}(t)$ становится зависимой также от константы вращательной диффузии частиц $D_{R}$ [13]. Как показала экспериментальная проверка, автокорреляционную функцию интенсивности для компоненты деполяризованного света $G_{H}^{V}(t)$, рассеянного частицами алмаза, можно связать только с их константами $D_{R}[14,15]$, заменив в соотношении (10) коэффициент $\tau_{1}$ на $\tau_{2}=6 D_{R}$, а автокорреляционную функцию $G_{V}^{V}(t)$ на $G_{H}^{V}(t)$.

Другой метод, более пригодный для определения распределения крупных частиц и агрегатов по размерам, основан на анализе кривых релаксации ЕО эффекта. В качестве ЕО эффекта выбран консервативный дихроизм $B$, который исследовался с помощью созданной авторами лабораторной установки. Такой дихроизм в основном определяется особенностями светорассеяния. Для определения релаксационных зависимостей $N(t)$ ЕО эффекта экспериментально определялись зависимости от времени интенсивностей света $J_{\|}(t)$ и $J_{\perp}(t)$, прошедшего сквозь дисперсную систему и поляризованного вдоль и перпендикулярно полю, создающему ориентационную упорядоченность частиц [16]. Зависимость $N(t)$ можно рассчитать с помощью соотношения

$$
N(t)=\ln \left(J_{\perp}(t) / J_{\|}(t)\right) .
$$

Теоретически релаксационную зависимость $N(t)$ можно представить формулой [17]

$$
N(t)=\int \exp \left(-t / \tau_{2}\right) \Delta K(r) \varphi(r) d r .
$$

Соотношение (11) было использовано для определения зависимости $f_{2}(r)=\Delta K(r) \varphi(r)$. Здесь $\Delta K(r)-$ зависимость дихроического веса от размеров частиц, и в случае белого света, как показала проверка [17], можно полагать, что зависимость $\Delta K(r)$ меняется пропорционально $r^{2}$ при размерах частиц, сопоставимых с длинами волн падающего света. Для малых частиц величина консервативного дихроизма мала. Вклад в $N(t)$ крупных частиц в основном определяется их рассеянием вперед, которое наиболее интенсивно. По этой причине области основного изменения $f_{2}(r)$ отвечают более крупные размеры частиц, чем области основного изменения $f_{1}(r)$. При ЕО исследованиях ориентационная упорядоченность частиц создавалась импульсами синусоидального поля частотой $10 \mathrm{kHz}$, напряженность которого не превышала $2 \mathrm{kV} / \mathrm{cm}$. Длительность импульсов поля менялось от 0.1 до $1000 \mathrm{~ms}$ и выбиралась в зависимости от размеров частиц и агрегатов из них.

\section{Системы для исследования}

В качестве дисперсной фазы для исследованных дисперсных систем использован порошок детонационного наноалмаза. Частицы дисперсной фазы получены детонационным методом и неоднородны по составу [18-23]. Часть частиц, образующихся в процессе синтеза, содержит кристаллический алмаз внутри и графитовую оболочку снаружи. Образуются также частицы графита и аморфного углерода, так как в процессе получения наноалмаза на стадии понижения температуры и давления часть алмаза может переходить в графит [19]. Дисперсная фаза наноалмаза состоит из первичных прочных агрегатов, в которых монокристаллы соединены $s p^{2}$-связями $[21,24,25]$. Агрегаты, так же как и монокристаллы алмаза, изотропны, а их показатель преломления близок к показателю преломления монокристаллов. Агрегаты, состоящие из жестко связанных более мелких частиц, можно уменьшить по размерам перемалыванием ультразвуковой обработкой, а также обработкой сильными кислотами при повышенных давлении и температуpe $[26,27]$. Окислением можно также удалять побочные продукты синтеза. Изотропные частицы графита также могут образоваться при синтезе. Они состоят из более мелких монокристаллов и имеют показатель преломления, близкий к показателю преломления алмаза [28,29].

Основная доля частиц дисперсной фазы содержала частицы с размерами менее $100 \mathrm{~nm}$, однако размеры отдельных частиц доходили до $400 \mathrm{~nm}$. Электронномикроскопический снимок частиц такой дисперсной фазы представлен на рис. 1. Здесь же представлена гистограмма распределения частиц по размерам частиц $d$ ( $d$ - среднеобъемный размер частицы).

При получении дисперсных систем для исследования вначале приготавливалась водная взвесь с объемной долей дисперсной фазы в несколько процентов. При седиментации частиц она разделилась на 8 фракций, различающиеся по цвету и имеющих четко выраженные тонкие границы. Нумерация фракций проводилась от верхнего слоя (с номером 1) к нижнему (с номером 8). Частицы фракций, входящих в разные слои, отличались по размерам и составу, а взаимодействуя при высоких их концентрациях, они образовывали рыхлые агрегаты 


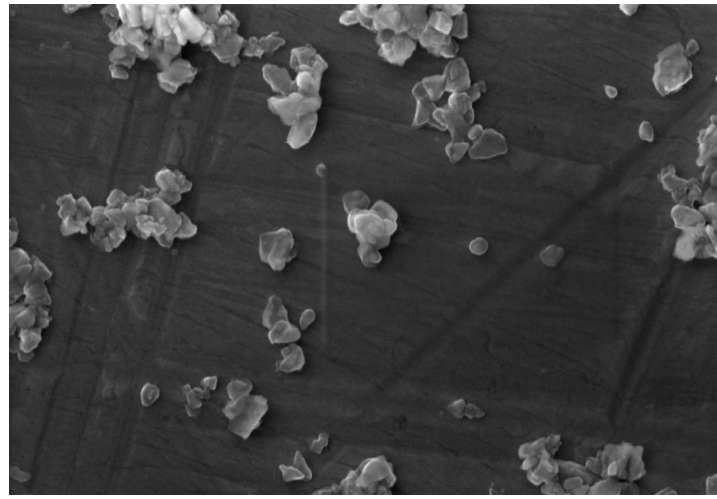

$-200 \mathrm{~nm}$

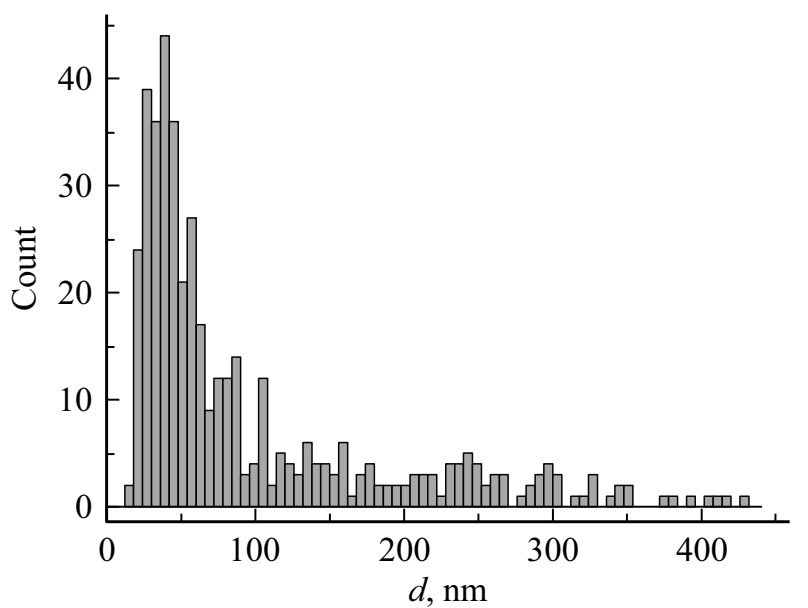

Рис. 1. Частицы дисперсной фазы.

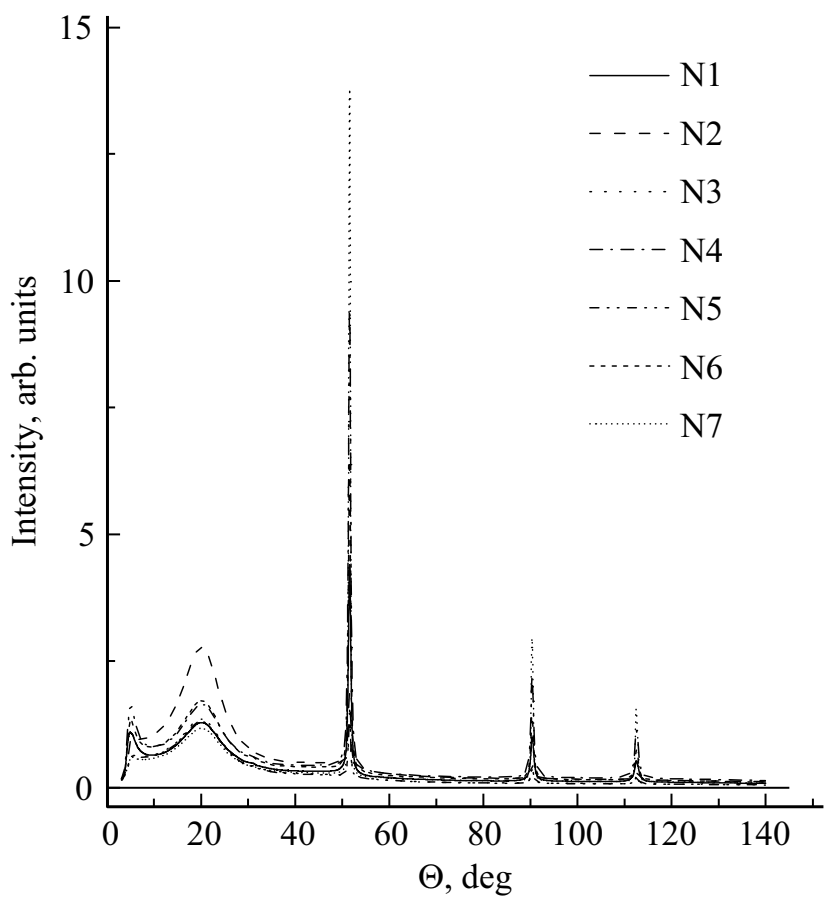

Рис. 2. Дифрактограмма дисперсной фазы.

фрактального типа. Рентгеноструктурный анализ подтвердил наличие поликристаллического алмаза и графита в дисперсной фазе всех полученных фракций. На рис. 2 представлены результаты рентгеноструктурного анализа образцов. Как видно из рисунка, для всех образцов в области углов дифракции $55^{\circ}, 92^{\circ}$ и $115^{\circ}$ наблюдаются пики, свойственные алмазу. Широкому пику с максимумом при $20^{\circ}$ соответствует поликристаллический графит. Этой же области могут соответствовать фулерены $\mathrm{C}_{60}$ и $\mathrm{C}_{70}$, но их доля очень мала.

Крупные частицы, делая системы мутными даже при незначительных их концентрациях, размывают световой луч, что существенно понижает допустимую для иссле- дований рефракции объемную долю дисперсной фазы. Чтобы получить системы, однократно рассеивающие свет, пригодные для измерения их показателя преломления и не коагулирующие в процессе исследований, дисперсные системы с частицами всех восьми фракций разбавлялись водой до концентраций, при которых объемная доля дисперсной фазы не превышала $0.01 \%$. Перед исследованиями полученные дисперсные системы встряхивались и подвергались ультразвуковой обработке.

\section{Результаты оптических исследований}

Для того чтобы оценить области изменения размеров частиц во фракциях DLS и EO методами определены автокорреляционные функции $G_{V}^{V}(t)$ при $\lambda=653 \mathrm{~nm}$ и кривые релаксации $N(t)$ в белом свете. Зависимости $f_{1}(r)$ и $f_{2}(r)$, рассчитанные как решения уравнений $(10)$ и (12) с экспериментально определенными левыми частями $G_{V}^{V}(t)$ и $N(t)$, представлены на рис. 3 .

Как видно из рисунка, для дисперсных систем N1-N6 зависимости $f_{1}(t)$ и $f_{2}(t)$ близки, а их максимумы удовлетворительно согласуются с максимумом гистограммы, представленной на рис. 1. Максимумы $f_{1}(r)$ и $f_{2}(r)$ для образцов N7 и N8 смещены в сторону более крупных размеров, что свидетельствует о присутствии агрегатов из частиц, более точно определяемых ЭО методом.

Были измерены значения показателей преломления $n$ при разных концентрациях частиц в приготовленных образцах, что позволило определить для них значения инкремента $d n / d \rho$. Значения $n$, измеренные всеми тремя указанными выше методами, совпали в пределах чувствительности использованных рефрактометров. Выбор максимально допустимой объемной доли частиц $(\Delta V / V)_{\max }$ зависел от мутности дисперсных систем, которая менялась немонотонно при переходе от слоя к слою. Для образца N8 было возможно проводить измерения показателя преломления только 

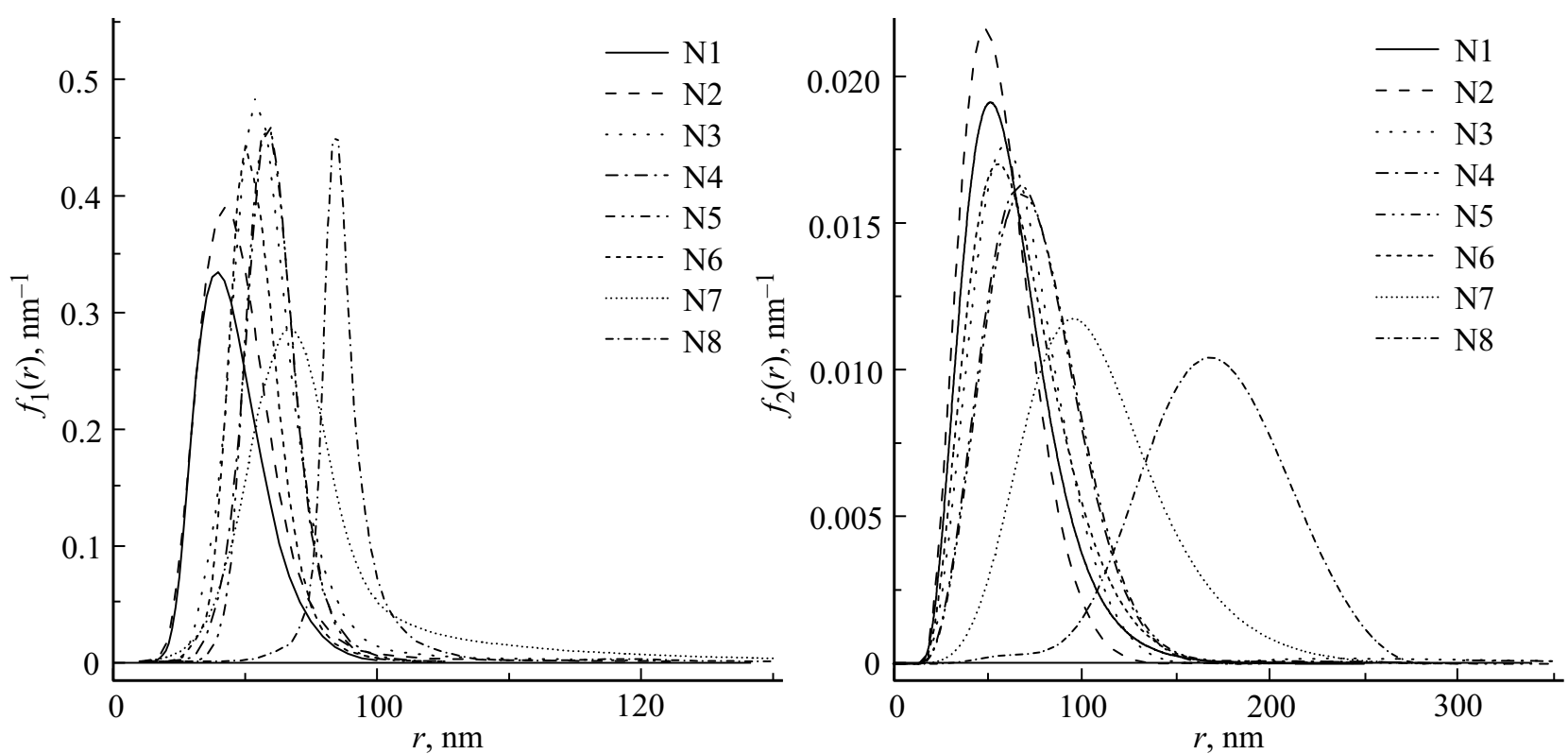

Рис. 3. Оценка полидисперсности согласно уравнениям (2) и (3). Функции $f_{1}(r)-$ слева, $f_{2}(r)-$ справа.

Таблица 1. Значения $d n / d \rho$ при $20^{\circ} \mathrm{C}$ и значения $(\Delta V / V)_{\max }$ для образцов $\mathrm{N} 1-\mathrm{N} 7$

\begin{tabular}{c|c|c|c|c|c|c|c}
\hline $\mathrm{N}$ образца & 1 & 2 & 3 & 4 & 5 & 6 & 7 \\
\hline$d n / d \rho$ & 0.49 & 0.44 & 0.45 & 0.44 & 0.48 & 0.44 & 0.43 \\
$(\Delta V / V)_{\max } \cdot 10^{4}$ & 3.3 & 1.9 & 0.66 & 3.5 & 0.8 & 2.5 & 0.46
\end{tabular}

при $(\Delta V / V)_{\max }=5 \cdot 10^{-6}$, так как он содержал крупные частицы и обладал высокой мутностью. Однако при столь низкой объемной доле частиц не удавалось надежно определить значение $d n / d \rho$. Удаление из образца крупных частиц и агрегатов из них позволяет повысить значение $(\Delta V / V)_{\max }$. Значения $d n / d \rho$ и $(\Delta V / V)_{\max }$ для образцов $\mathrm{N} 1-\mathrm{N} 7$ представлены в табл. 1.

Мутность и значения $(\Delta V / V)_{\max }$ для этих образцов существенно различались, а значения $d n / d \rho$ близки. Это позволяет предположить, что во всех этих образцах разность $n-n_{0}$ определяется близкими по структуре мелкими частицами, которые составляют основную долю дисперсной фазы. Более крупные частицы и агрегаты, определяющие мутность систем, слабо влияют на их показатель преломления. Удаление из исследованных дисперсных систем крупных частиц и агрегатов центрифугированием слабо влияло на их плотность и показатель преломления, но существенно понижало их мутность. После центрифугирования всех образцов в центробежном поле $15000 \mathrm{~g}$ в течение $5 \mathrm{~min}$ в них оставались только частицы с радиусами $r<35 \mathrm{~nm}$, если их плотность близка к плотности алмаза, и $r<50 \mathrm{~nm}$, если их плотность близка к плотности графита. Измеренные значения $d n / d \rho$ центрифугированных образцов NN 1, 4 и 7 равны соответственно $0.38,0.34$ и 0.31 . Более длительное центрифугирование несколько повы- шало значение $d n / d \rho$, что свидетельствовало о том, что уменьшение размеров частиц увеличивает их влияние на показатель преломления дисперсных систем. Так, центрифугирование образца $\mathrm{N} 4$ в течение $1 \mathrm{~h}$ в поле $15000 \mathrm{~g}$ удаляло из него частицы с радиусом более $12 \mathrm{~nm}$, если полагать, что это частицы алмаза, и $17 \mathrm{~nm}$, если полагать, что это частицы графита, а $d n / d \rho$ такой системы возрастает до значения 0.42. Это согласуется с тем, что мелкие частицы вносят больший вклад в показатель преломления дисперсной системы, чем крупные частицы.

Как отмечено выше, дисперсная фаза исследованных дисперсных систем состоит из поликристаллических алмаза, графита, а также иных малых добавок, которые мы здесь не учитывали. Действительная часть показателя преломления графита [28] близка к показателю преломления алмаза, и удельная рефракция дисперсной фаза $R_{p}$ слабо зависит от долей в ней алмаза и графита. Так, рассчитанные для алмаза и графита значения удельной рефракции равны соответственно 0.61 и 0.67 . При расчетах полагалось, что дисперсионная среда имеет плотность воды. В этом случае значение $\rho_{p}-\rho_{0}$ равно 2.52 для алмаза, а для графита оно может меняться в пределах 1.2-1.23.

Если использовать при расчетах инкремента показателя преломления соотношения (4) и (5), соответствующие молекулярно-оптическому подходу, то для водной дисперсной системы алмаза $d n / d \rho=0.21$, а для графита $d n / d \rho=0.48$. Это соответствует тому, что в частицы всех исследованных систем входит как алмаз, так и графит. Так как в детонационный алмаз входит графит, то его доля в дисперсной фазе центрифугированных в течение 5 min образцов N1, N4 и N7 составляет соответственно 43,40 и $39 \%$, что согласуется с литературными данными $[19,20]$. 


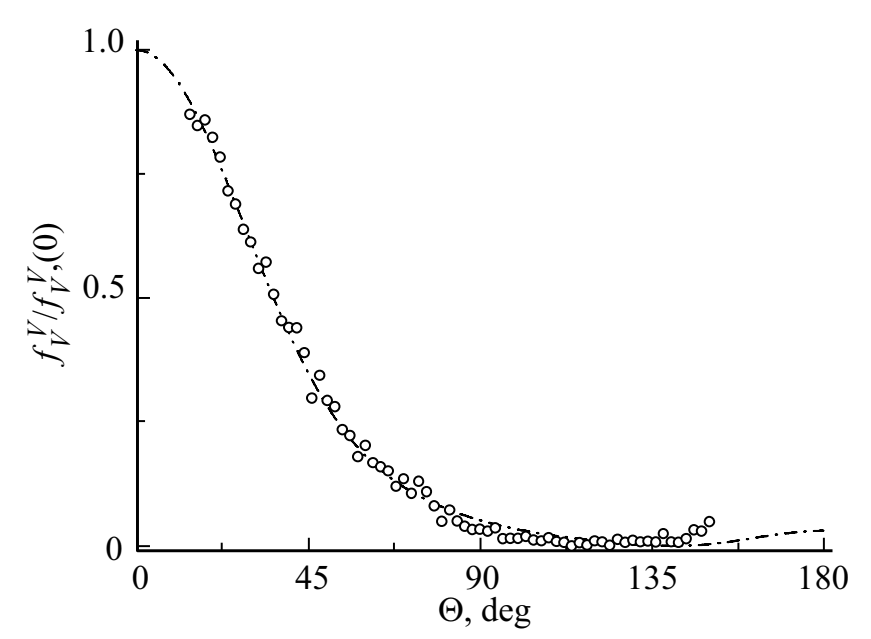

Рис. 4. Индикатриса светорассеяния при $\lambda=657 \mathrm{~nm}$. Сплошная кривая - расчет, точки - эксперимент.

Таблица 2. Значения $d n / d \rho$, рассчитанные согласно теории Ми

\begin{tabular}{c|c|c|c|c}
\hline $\begin{array}{c}\mathrm{N} \\
\text { образца }\end{array}$ & $\begin{array}{c}\text { Графит } \\
(\mathrm{DLS})\end{array}$ & $\begin{array}{c}\text { Графит } \\
(\mathrm{EO})\end{array}$ & $\begin{array}{c}\text { Алмаз } \\
(\mathrm{DLS})\end{array}$ & $\begin{array}{c}\text { Алмаз } \\
(\mathrm{EO})\end{array}$ \\
\hline 1 & 0.48 & 0.40 & 0.52 & 0.48 \\
4 & 0.45 & 0.42 & 0.45 & 0.45 \\
7 & 0.45 & 0.38 & 0.51 & 0.40
\end{tabular}

Согласно теории рассеяния, в отличие от молекулярно-оптической теории инкремент показателя преломления дисперсных систем зависит от размеров и формы частиц [7]. Соотношения (7)-(9) получены для систем, содержащих сферические частицы. Для того чтобы определить, в какой степени они применимы к дисперсным системам алмаза, были проведены иссле- дования их статического рассеяния. Так как частицы алмаза имеют несферическую форму, степень деполяризации $\Delta_{v}$ света, рассеянного дисперсными системами с такими частицами, отлична от нуля. Однако, как показал эксперимент, она мала и не превышает 7\% во всем диапазоне углов рассеяния $\theta$. Как отмечалось ранее, угловая зависимость интенсивности рассеянного света $I_{V}^{V}$, соответствующая поляризации падающего и рассеянного света ортогонально плоскости рассеяния, соответствует рассеянию света сферами [15]. На рис. 4 сопоставлены экспериментальная и рассчитанная в рамках теории Ми зависимости $I_{V}^{V}$ для разбавленной системы, распределение частиц по размерам в которой соответствует гистограмме, представленной на рис. 1. Основному ее изменению соответствуют малые углы рассеяния, что согласуется с зависимостями $I_{V}^{V}(\theta)$ частиц, соизмеримых с длиной световой волны.

Близость экспериментальной и теоретической индикатрис допускает использование соотношений (7) и (8) при исследовании рефракции дисперсных систем алмаза. Зависимости $d n / d \rho$ и $d n^{\prime} / d \rho$ от размеров $r$ частиц алмаза и графита, рассчитанные в рамках теории Ми по формулам (7) и (8) при $\lambda=657 \mathrm{~nm}$, представлены на рис. 5.

При расчетах полагалось, что для алмаза $n_{p}=2.42$ и для графита $n_{p}=2.68$. Для мнимых частей алмаза и графита использованы значения 0 и 1.39 соответственно [28,29].

Как видно из рисунка, показатель преломления дисперсной системы алмаза слабо зависит от размеров частиц в области $30<r<110 \mathrm{~nm}$, и дальнейшее увеличение размеров частиц при сохранении их объемной доли должно приводить к резкому понижению показателя преломления дисперсной системы, а при $r>225 \mathrm{~nm}$ он становится даже меньше показателя преломления дисперсионной среды. Если полагать что функции распределения $\varphi(r)$ для исследованных образцов определяются

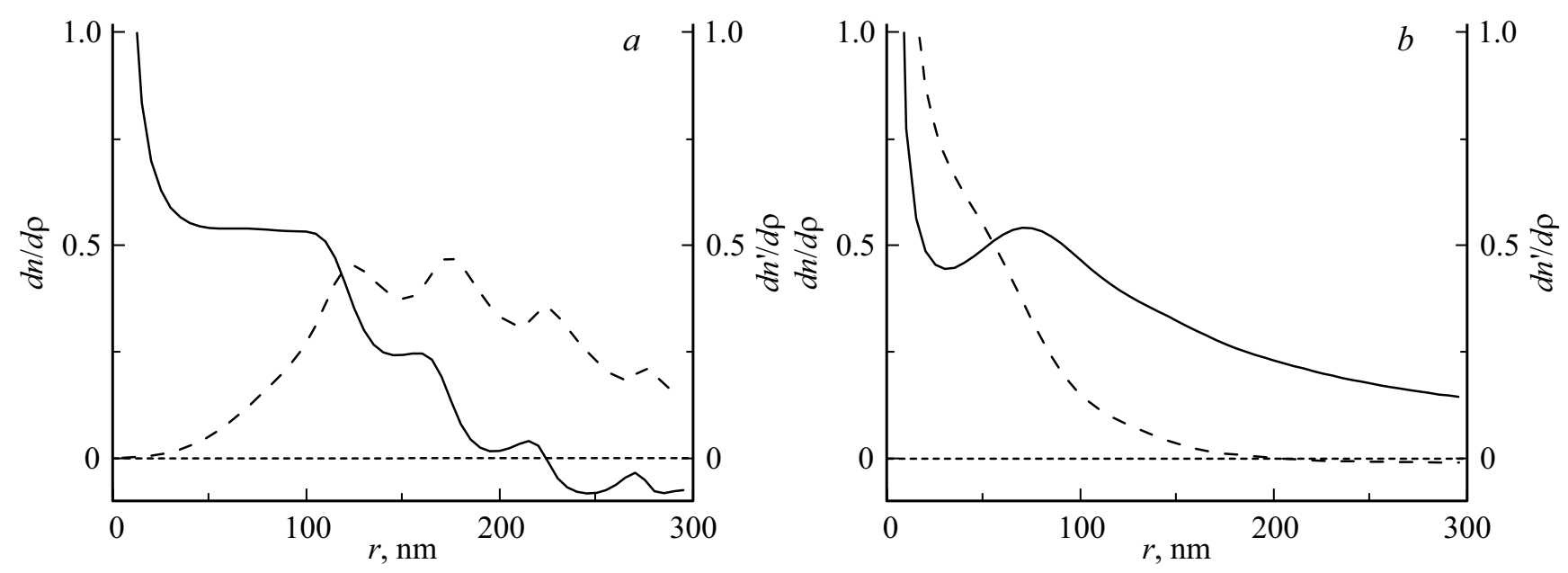

Рис. 5. Зависимости $d n / d \rho$ (сплошная кривая) и $d n^{\prime} / d \rho$ (штриховая) водных дисперсных систем алмаза $(a)$ и графита $(b)$ от радиуса сферических частиц дисперсной фазы. 
DLS или ЕО методами, то значения $d n / d \rho$ для частиц, состоящих из алмаза и графита, близки между собой, но несколько превышают экспериментально измеренные значения. Для образцов N1, N4 и N7 рассчитанные значения $d n / d \rho$ представлены в табл. 2 .

Образованию агрегатов из частиц должно соответствовать увеличение размеров $r$ при сохранении объемной доли $\Delta V / V$. Согласно представленным на рис. 4 зависимостям $d n^{\prime} / d \rho$ от $r$, дисперсные системы с частицами графита при образовании агрегатов должны просветляться, а алмаза становиться более мутными при $r<150 \mathrm{~nm}$. Значение $d n^{\prime} / d \rho$, определяющее мутность дисперсных систем алмаза, велико при $r>50 \mathrm{~nm}$. В этой области размеров частиц проходящий сквозь коллоид световой луч не размывается только при крайне низкой объемной доле $\Delta V / V$ частиц в дисперсной системе. Согласно расчетам, показатель преломления системы алмаза с частицами, соизмеримыми с длиной световой волны, в эксперименте не должен отличаться от показателя преломления дисперсионной среды. Этот вывод подтверждается ранее проведенными экспериментальными исследованиями показателя преломления дисперсных систем [4].

\section{Выводы}

1. По результатам исследования водных дисперсных систем детонационного алмаза можно заключить, что основной вклад в инкремент показателя преломления вносят частицы с размерами менее $20 \mathrm{~nm}$, а крупные частицы слабо влияют на рефракцию систем.

2. При расчете показателя преломления этих систем применим молекулярно-оптический метод расчета показателя преломления растворов, что позволяет определять долю алмаза и графита в дисперсной фазе.

3. Результаты расчета показателя преломления систем с частицами алмаза и графита в рамках теории рассеяния Ми несколько выше полученных экспериментально при размерах частиц менее $100 \mathrm{~nm}$.

Работа выполнена при поддержке гранта РФФИ 1603-00495А. Часть исследований выполнена в „Междисциплинарном ресурсном центре по направлению Нанотехнологии“ и „Центре диагностики функциональных материалов для медицины, фармакологии и наноэлектроники“ ресурсного парка Санкт-Петербургского государственного университета.

\section{Список литературы}

[1] Иофбе Б.В. Рефрактометрические методы химии. Л.: Химия, 1974. 399 с.

[2] Волкенштейн М.В. Молекулы и их строение. М.-Л.: Издво АН СССР, 1955. 229 c.

[3] Кройт Г.Р. Наука о коллоидах. М.: Ин. лит., 1955. 540 с.

[4] Войтылов В.В., Петров М.П., Спартаков А.А., Трусов А.A. // Опт. и спектр. 2013. Т. 114. № 4. С. 687.
[5] Вукс М.Ф. Электрические и оптические свойства молекул и конденсированных сред. Л.: Изд-во Ленинградского университета. 1984. $336 \mathrm{c}$.

[6] Шибрин К.С. Рассеяние света в мутной среде. М.-Л.: Госизд-во техн.-теор. лит., 1951. 288 с.

[7] Ван де Хюлст $Г$. Рассеяние света малыми частицами. М.: Ин. лит., 1961. 537 c.; Van de Hulst H.C. Light Scattering by Small Particles. NY: John Wiley \& Sons, 1957. 470 p.

[8] Mishchenko M.I., Dlugach J.M., Yurkin M.A., Bi L., Cairns B., Liu L., Panettae R.L., Travis L.D., Yang P., Zakharova N.T. // Physics Reports. 2016. V. 632. P. 1.

[9] Mie G. // Ann. Phys. 1908. V. 330. P. 377. doi 10. 1002/andp.19083300302

[10] Reyes Coronado A., Garsia-Vlenzuela A., Sanchez-Perez C., Barrera R.G. // J. Phys. 2005. V. 7. P. 89. doi 10.1088/13672630/7/1/089

[11] Garcia-Valenzuela A., Barrera R.G., Gutierrez Reyes E. // Optics Express. 2008. V. 16. P. 19743. doi 10.1364/OE.16.019741

[12] Berne B.J., Pecora R. Dynamic Light Scattering with Application to Chemistry, Biology and Physics. NY.: Dover Publication, 2000. $371 \mathrm{p}$.

[13] Aleksenskii A.E., Shvidchenko A.V., Eidel'man E.D. // Technical Physics Letters. 2012. V. 38. P. 1049.

[14] Melnikov A., Spartakov A., Trusov A., Vojtylov V. // Colloids and Surfaces. B 2007. V. 56. P. 65.

[15] Klemeshev S.A., Petrov M.P., Rolich V.I., Trusov A.A., Voitylov A.V., Vojtylov V.V. // Diamond and Related Materials. 2016. V. 69. P. 177. doi 10.1016/j.diamond.2016.08.016

[16] Klemeshev V.A., Voitylov A.V., Klemeshev S.A., Petrov M.P. // Intern. Conf. on Computer Technologies in Physical and Engineering Applications (ICCTPEA). St. Petersburg, Russia, 2014. P. 181.

[17] Trusov A., Vojtylov V. // Electrooptics and Conductometry of Polydisperse Systems. NY: CRC Press, 1993. 145 p.

[18] Vul' A.Y., Eydelman E.D. Detonation Nanodiamonds: Science and Applications. / Ed. by Vul' A.Y., Shenderova O.V. 2014.

[19] Katherine B Holt. Diamond at the Nanoscale: Applications of Diamond Nanoparticles from Cellular Biomarkers to Quantum Computing. 2007. doi 10.1098/rsta.2007.0005

[20] Dolmatov V.Yu. // Russ. Chem. Rev. 2007. V. 76 (4). P. 339. doi https://doi.org/10.1070/RC2007v076n04ABEH003643

[21] Dolmatov V.Y., Veretennikova M.V., Marchukov V.A., Sushchev V.G. // Phys. Sol. State. 2004. V. 46. P. 611.

[22] Grichko V., Tyler T., Grishko V.I., Shenderova O. // Nanotechnology. 2008. V. 19. N 22. P. 225201.

[23] Osawa E. // Pure and Applied Chemistry. 2008. V. 80. N 7. P. 1365.

[24] Chukhaeva S.I. // Physics of the Solid State. 2004. V. 46. N 4. P. 625.

[25] Williams O.A., Hees J., Dieker C., Jäger W., Kirste L., Nebel C.E. // ACS Nano. 2010. V. 4. N 8. P. 4824.

[26] Fedutik Y.A., Antipov A.A., Maltseva E.A., Kalachev A.A. // 3rd International Symposium „Detonation Nanodiamonds: Technology, Properties and Applications“. St. Petersburg, Russia, 2008. P. 65-72.

[27] Pichot V., Comet M., Fousson E., Baras C., Senger A., Le Normand F., Spitzer D. // Diamond and Related Materials. 2008. V. 17. N 1. P. 13.

[28] Djurišić A.B., Li E.H. // J. Appl. Phys. 1999. V. 85. P. 7404.

[29] Stagg B.J., Charalampopoulos T.T. // Combustion and Flame. 1993. V. 94. P. 381. 